\begin{tabular}{|c|l|}
\hline Title & A llometry in Phy sarum plasmodium during free locomotion: size versus shape, speed and rhythm \\
\hline Author(s) & Kuroda, Shigeru; Takagi, Seiji; Nakagaki, Toshiyuki; U eda, Tetsuo \\
\hline Citation & $\begin{array}{l}\text { Journal of experimental biology, 218(23), 3729-3738 } \\
\text { https://doi.org/L0.1242/3eb.124354 }\end{array}$ \\
\hline Issue Date & 2015-12-01 \\
\hline Doc URL & http://hdl.handle.net/2115/63698 \\
\hline Rights & ○ 2015. Published by The Company of Biologists Ltd \\
\hline Type & article \\
\hline File Information & 3729.full.pdf \\
\hline
\end{tabular}

Instructions for use 


\title{
Allometry in Physarum plasmodium during free locomotion: size versus shape, speed and rhythm
}

\author{
Shigeru Kuroda ${ }^{1, *}$, Seiji Takagi ${ }^{2}$, Toshiyuki Nakagaki ${ }^{1}$ and Tetsuo Ueda ${ }^{1}$
}

\begin{abstract}
Physarum plasmodium is a giant unicellular organism whose length can vary by more than three orders of magnitude. Using plasmodia ranging in size from $100 \mu \mathrm{m}$ to $10 \mathrm{~cm}$, we investigated the size dependency of their thickness distributions and locomotion speeds during free locomotion. (1) In the longitudinal direction, the organism is thickest close to the front, and decreases exponentially in thickness towards the rear. The slenderness ratio varies with body size according to a power law, such that large plasmodia are long and flat, whereas small plasmodia are short and thick. (2) The mean locomotion speed is proportional to the mean maximum thickness of the frontal part. By conducting a dimensional analysis, possible physical models are discussed. (3) The intrinsic period of the thickness oscillation, which is related to shuttle streaming (period 1-2 min), increases logarithmically with body size. (4) Various

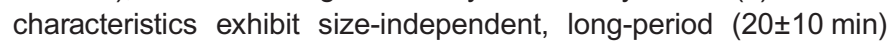
oscillations, including speed, shape and intrinsic thickness oscillation period. These variations are closely coupled to formation of the entire cell shape, including undulation of thickness along the longitudinal axis and timing of branching of the frontal tip. Based on these experimental results and those reported previously, we propose a simple mathematical model for cell locomotion.
\end{abstract}

KEY WORDS: Cell locomotion, Scaling, Self-organization, Dimensional analysis, Spatial memory, Localization of locomotion machinery, Traveling wave

\section{INTRODUCTION}

Biological scaling, referred to as allometry, is of great importance because it does not involve chance but instead tells us about the physical laws behind biological behaviour (Shingleton, 2010; Calder, 1996; Schmidt-Nielsen, 1984; McMahon and Bonner, 1983; Barenblatt, 2003). In particular, the observation of scaling laws that hold across size differences of several orders of magnitude may lead to substantial revisions in the understanding not only of the field of interest, but also many other fields of study. However, data involving large size differences are usually gathered from many animal species because size differences are usually limited in a single species. The plasmodium of the true slime mould Physarum polycepharum is an exception and serves as an ideal candidate for allometric studies because of its variation in size over several orders of magnitude.

The plasmodium of true slime mould is a multinuclear single-cell organism and moves like an amoeboid cell. Its size varies from $10^{-5}$

\footnotetext{
${ }^{1}$ Research Institute for Electric Science, Hokkaido University, N20W10, Kita-ku, Sapporo, Hokkaido 001-0020, Japan. ${ }^{2}$ The School of Systems Information Science, Future University Hakodate, 116-2 Kamedanakano-cho, Hakodate, Hokkaido 041 8655, Japan.

*Author for correspondence (shigeru.kuroda@es.hokudai.ac.jp)
}

Received 1 May 2015; Accepted 22 September 2015 to $10^{0} \mathrm{~m}$. Nuclear division occurs synchronously without cell division every $10 \mathrm{~h}$ (Ashworth and Dee, 1975). Two plasmodia with the same genetic fusion type can coalesce to form a single plasmodium. Conversely, a single plasmodium can be divided into many parts, each of which eventually forms a new, tiny plasmodium. This provides a convenient experimental way to generate cells with sizes varying over several orders of magnitude. Body size can thus be regarded as a system parameter that can be varied, inducing quantitative and/or qualitative changes in other characteristics of the plasmodium.

It is known that cells serve not only as the fundamental unit for the engagement of life activity but also have the ability to discriminate their environment, to make decisions and to behave appropriately. The shape of an amoeboid cell, including plasmodium, is very flexible and has essentially infinite degrees of freedom. However, specific situations place restrictions on these degrees of freedom such that the organism self-organises its shape in order to remain functional.

Directional movement is one such situation. For example, if an extracted protoplasm droplet or a small piece taken from a large plasmodium is placed on plain agar gel, it begins to shape its body after a given time, and finally migrates in a specific direction (Fig. 1A-F). The plasmodia shown in Fig. 2 all migrate in the upper left direction. Smaller plasmodia adopt tadpole-like shapes (Fig. 2A,B). As they become larger, the extended edges of the plasmodia tend to branch (Fig. 2C) and their two-dimensional shape increases in complexity. Finally, very large migrating plasmodia (in the order of a centimetre) extend two-dimensionally to adopt a fanlike shape with a complicated network of veins towards the rear (Fig. 2D). It is empirically believed that the longitudinal distribution of thickness during unidirectional locomotion has a similar tendency among plasmodia of various sizes. The thickness is high close to the leading edge and gradually decreases towards the rear. Therefore, the direction of migration can always be predicted from the distribution of the protoplasm. However, there are no quantitative reports on the relationship between the longitudinal thickness distribution and cell size.

It is also well known that large plasmodia tend to move faster than small plasmodia. However, except for one brief report (Ueda, 2006), to our knowledge there are no quantitative studies on the relationship between locomotion speed and cell size.

Plasmodia exhibit a coordinated, regular oscillation in thickness with a period of 1-2 min (longer in large plasmodia than in small plasmodia) that involves protoplasmic flow inside the body. It is also empirically known that the contraction period in large plasmodia is longer than that in small plasmodia. This phenomenon was first observed by Vouk (1910) at the beginning of last century, and there are some quantitative reports on small plasmodia with cell sizes limited to less than $1 \mathrm{~mm}$ (Ueda and Kobatake, 1982; Ueda and Kobatake, 1980).

Here, we report on a comprehensive allometric analysis of three types of characteristics (shape, speed and rhythm) in 


\begin{tabular}{|c|c|}
\hline \multicolumn{2}{|c|}{ List of symbols and abbreviations } \\
\hline$D_{\mathrm{C}}$ & diffusion level of a chemical C \\
\hline$h_{\max }(t)$ & maximal thickness $(\mathrm{mm})$ \\
\hline longitudinal shape & $\begin{array}{l}\text { the contour of thickness distribution of the } \\
\text { plasmodium in the longitudinal direction } \\
\text { during one-dimensional locomotion }\end{array}$ \\
\hline$r$ & $\begin{array}{l}\text { Pearson's correlation coefficient after the } \\
\text { logarithmic transformation }\end{array}$ \\
\hline$t$ & time \\
\hline$T_{0}(t)$ & $\begin{array}{l}\text { intrinsic period of the thickness oscillation } \\
\text { related to shuttle streaming (s) }\end{array}$ \\
\hline$T_{1}(z)$ & $\begin{array}{l}\text { period of the large, long-term variation of a } \\
\quad \text { variable } z(t) \in\left\{h_{\max }, T_{0}, v\right\} \text { (min) }\end{array}$ \\
\hline V & volume $\left(\mathrm{mm}^{3}\right)$ \\
\hline$v(t)$ & locomotion speed $\left(\mathrm{mm} \mathrm{min}^{-1}\right)$ \\
\hline$V_{/}(t)$ & volume per unit width $\left(\mathrm{mm}^{3} \mathrm{~mm}^{-1}\right)$ \\
\hline$w_{\text {mean }}(t)$ & mean width of a plasmodium (mm) \\
\hline$x_{\mathrm{L}}(t)$ & $\begin{array}{l}\text { distance from the leading edge to the tail } \\
\text { edge }(\mathrm{mm})\end{array}$ \\
\hline$x_{\max }(t)$ & $\begin{array}{l}\text { distance from the frontal tip to the position } \\
\text { of maximum thickness }(\mathrm{mm})\end{array}$ \\
\hline$\alpha$ & $\begin{array}{l}\text { exponent in the exponential function } \\
\beta \exp \left[-\left(x-x_{\max }\right) / \alpha+\gamma\right] \text {, referred to as the } \\
\text { 'exponential decay distance' (mm) }\end{array}$ \\
\hline$\tau^{*}(x, y)$ & $\begin{array}{l}\text { time-lag maximising the correlation } \\
\text { between } x(t+\tau) \text { and } y(\tau) \text { where } \\
x, y \in\left\{h_{\max }, T_{0}^{-1}, v\right\} \text { (min) }\end{array}$ \\
\hline
\end{tabular}

plasmodia with sizes ranging from $100 \mu \mathrm{m}$ to $10 \mathrm{~cm}$, that is, for plasmodia differing by three orders of magnitude in length during free locomotion. For each plasmodium, we obtained one-dimensional locomotion data over the course of $6-8 \mathrm{~h}$. We observed well-defined allometric relations with the shape and locomotion speed and discuss the physical basis of our findings using dimensional analysis. Although we observed size-dependent properties in the intrinsic thickness oscillation period, we also identified significant long-term variations, which were independent of size. Such long-term variation was also evident for the locomotion speed and shape. We show that the evolution of body shape is tightly coupled with these long-lasting variations. Two examples are presented: (1) undulations in the longitudinal distribution of thickness and (2) the timing of branching of the frontal part of migrating plasmodia. Both are discussed from the viewpoint of the dynamic encoding of temporal information to the spatial structure.
A simple mathematical model describing this type of phenomenon is then presented.

\section{MATERIALS AND METHODS}

\section{Organism and culture}

The plasmodium of Physarum polycephalum (strain HU195×HU200) (Akitaya et al., 1985) was fed with oat flakes (Quaker Oats Co.) on agar gel at $24^{\circ} \mathrm{C}$ in the dark (Ueda et al., 1975). Agar gels used for all experiments were nutrient free (Wako Pure Chemicals Inc.) and their concentrations were fixed at $1.0 \%$ weight by volume.

\section{Preparation of plasmodia with various sizes using giant plasmodia}

Before experimental use, the organism was allowed to move on plain agar gel overnight. Using the frontal part, various sizes of plasmodia $(N=54)$ were prepared as described previously by Ishigami et al. (1987) for smaller sizes [volume $<0.1 \mathrm{~mm}^{3}(N=18)$ ] and Takagi et al. (2007) for larger sizes [volume $>0.1 \mathrm{~mm}^{3}(N=36)$ ]

The smaller plasmodia were placed on plain agar gel in Petri dishes $(9 \mathrm{~cm}$ in diameter) and were allowed to move freely. After a few hours, the organisms exhibited locomotion that was almost one-dimensional in nature and adopted tadpole-like shapes (Fig. 2A,B). Larger plasmodia had more than one advancing front (Fig. 2C), and still larger organisms adopted a fan-like shape with a sheet-like leading edge (Fig. 2D). Therefore, in order to limit the number of advancing fronts to one or to confine locomotion to one dimension, the larger plasmodia were placed on narrow agar gel lanes [width $=1.0(N=3), 1.5$ $(N=4), 4.0(N=24)$ or $8(N=5) \mathrm{mm}$ and length=17 or $25 \mathrm{~cm}]$, which were separated from the adjacent lanes using PET film (100 $\mu \mathrm{m}$ thickness) placed on the agar gel. These plasmodia were also allowed to move freely and after a few hours they exhibited stable, almost one-dimensional locomotion with shapes distributed in the longitudinal direction of the lane and a sheet-like leading edge (Fig. 2E) (Takagi et al., 2007). The experiments were conducted at room temperature $\left(24 \pm 1^{\circ} \mathrm{C}\right)$ and the humidity inside the apparatus was maintained at around $90 \%$ relative humidity $(\mathrm{RH})$ using a plastic lid that was lifted a little. The small plasmodia used in the study of branching timing were separately prepared from protoplasm droplets to control their body size (Takagi and Ueda, 2008). Their free migration on agar gel was then observed.

\section{Measurements of thickness of plasmodia by image analysis}

The plasmodia were illuminated from below using an infrared beam $(\lambda \sim 950 \mathrm{~nm})$ that was passed through light diffuser plates and viewed from above (Takagi et al., 2007). The transmitted light images were magnified using either a lens (NIKKOR-H Auto $28 \mathrm{~mm}$ f/3.5; Nikon or $55 \mathrm{~mm}$ telecentric f/2.8; Computar) or a microscope (C-DSD115; Nikon) and captured using a CCD camera (XC-ST70; Sony) or a cooled CCD camera (Retiga EXi Fast 1394 Mono Cooled; QImaging Corp.). They were stored in a personal computer as time-lapse images covering approximately $12 \mathrm{~h}$, where the time-lapse interval periods were $12(N=45), 20(N=3)$ and $30 \mathrm{~s}$
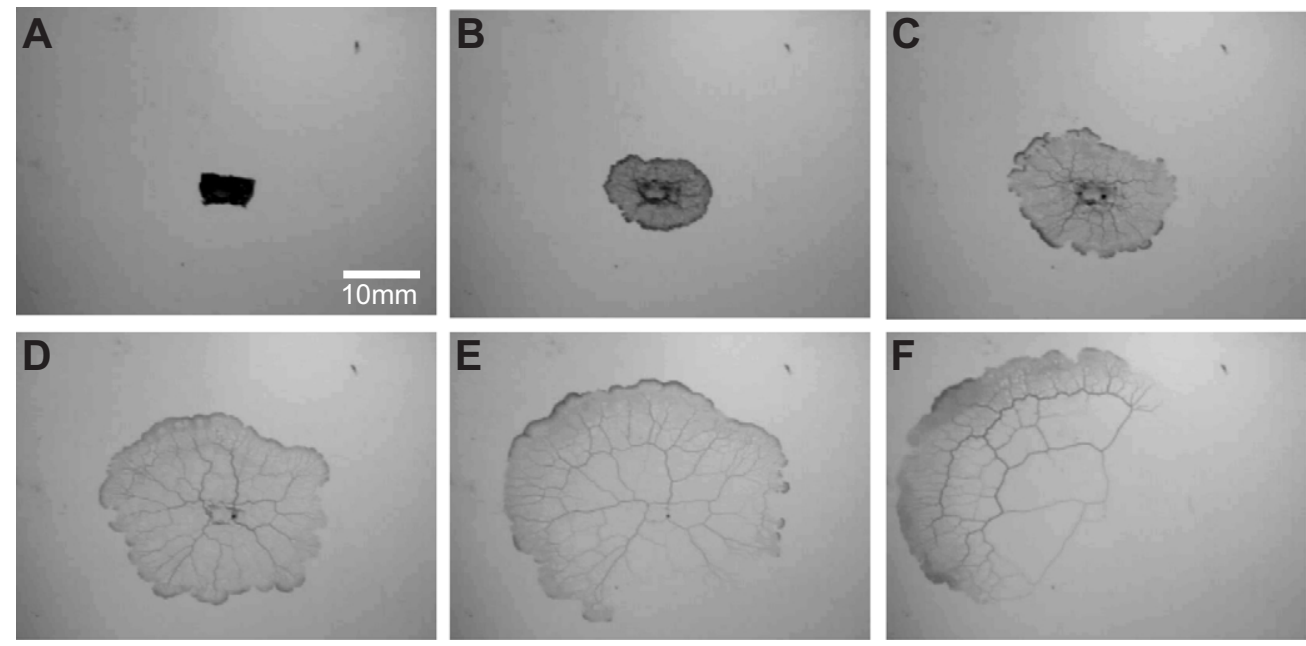

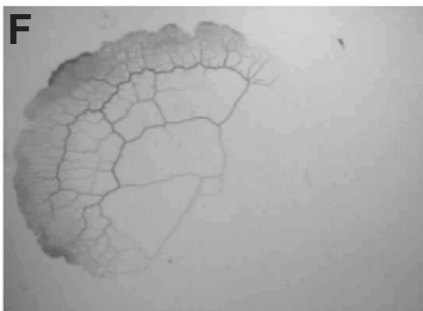

Fig. 1. Emergence of one-directional migration from Physarum polycephalum protoplasm.

(A-D) Images of the plasmodium, which was extending in almost all directions, after $0,150,300$ and $450 \mathrm{~min}$, respectively. $(E, F)$ Images of the plasmodium, which kept migrating in the upper left direction, after 600 and $810 \mathrm{~min}$, respectively. Darker contrast indicates areas with relatively thicker plasmodium. 


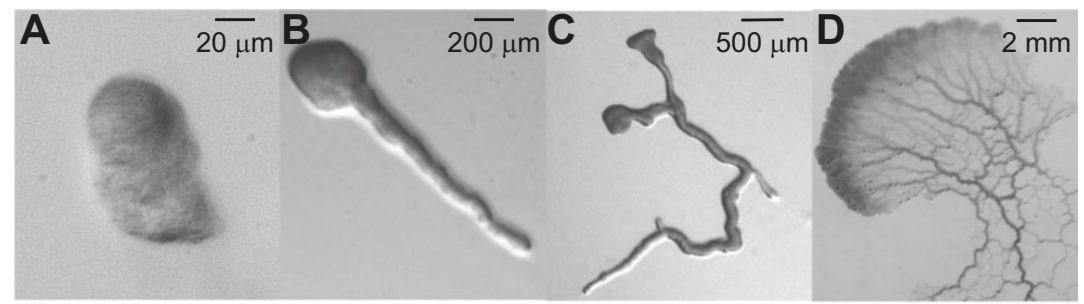

Fig. 2. Physarum polycephalum plasmodia during free locomotion. (A-D) Plasmodia of various sizes.

(E) Plasmodia migrating along narrow lanes.

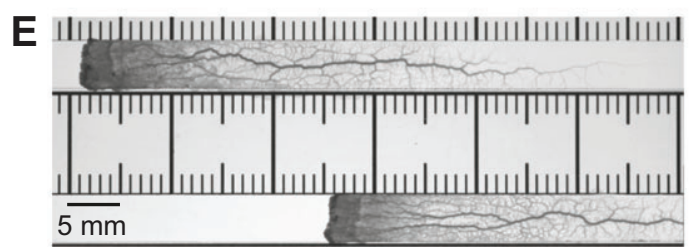

$(N=6)$. The spatial resolution of the images were $0.008-0.027 \mathrm{~mm}^{-1 x} \mathrm{pl}^{-1}$ for the smaller plasmodia and $0.072-0.363 \mathrm{~mm}$ pixel $^{-1}$ for the larger plasmodia. The field of view was $720 \times 480$ or $696 \times 520$ pixels.

Pixel values in the images were converted to optical density (OD) values, which were calculated as $-\log \left(I / I_{0}\right)$, where $I$ and $I_{0}$ are the transmitted and incident light intensities, respectively. A calibration table relating the OD to the thickness of the plasmodium was constructed for each measurement apparatus by confining the protoplasmic sol in glass tubes with various known diameters (Takagi and Ueda, 2008) or by placing the protoplasm between a glass slide and a glass cover with a small tilt angle. The thickness of the plasmodium under study was linearly interpolated from these calibration tables.

Thus, the thickness of a plasmodium $h(x, t)$ was obtained as a function of position $x$ and time (or frame) $t$. We analysed the resulting spatiotemporal patterns using programs that we developed in-house as plug-in software for ImageJ (Rasband, 2011).

\section{Data analysis}

\section{Longitude coordinate and measurement of locomotion speed}

The plasmodia exhibited stable locomotion in an almost one-dimensional manner. However, in experiments using tiny plasmodia and no lane, it was occasionally (once or twice per recording) observed that the plasmodium changed direction sharply ( $>90 \mathrm{deg}$ ) or flipped its front and tail. Such frames were excluded from further analysis. For each plasmodium, we obtained one-dimensional locomotion data over the course of 6-8 h. The mean values of various characteristics (averaged over the measurement time) were examined and denoted by a bar over the symbols. To analyse the directional movement of a plasmodium, we introduced a longitude curve roughly coinciding with the centre line of the trail and also containing the frontal tip and body axis as far as possible in every frame (Fig. 3A). The longitude curve and its perpendicular lines are referred to as the longitude coordinate for locomotion, which is used to describe the shape and movement of the plasmodium. The coordinates of the leading edge $x_{f}(t)$ and tail edge $x_{b}(t)$ on the longitude axis were determined for each frame $t$ using a threshold criterion (Fig. 3A). The locomotion speed of the plasmodium was calculated from the distance moved by the leading edge along the longitude axis during a fixed time interval $\left(\Delta_{t}=4\right.$ or $\left.8 \mathrm{~min}\right)$. We refer to points lying on the longitude axis between $x_{f}(\mathrm{t})$ and $x_{b}(t)$ as 'body points' in the following analysis. To outline the body area of the plasmodium in each frame, for each body point $x$, a connected body component $l(x, t)$ on the perpendicular axis centred at $x$ was determined using a threshold criterion; the width of the plasmodium $w(x, t)$ was obtained as the length of $l(x, t)$; the maximum width and the mean width, were given by:

$$
\begin{aligned}
& w_{\text {max }}(t)=\max _{x_{b}(t) \leq x \leq x_{f}(t)} w(x, t) \text { and } \\
& w_{\text {mean }}(t)=\int_{x_{b}(t)}^{x_{f}(t)} w(x, t) \mathrm{d} x /\left(x_{f}(t)-x_{b}(t)\right),
\end{aligned}
$$

respectively. The volume $V$ of the plasmodium was estimated by body area $\times$ thickness, such that:

$$
V=\int_{x_{b}(t)}^{x_{f}(t)} \int_{y \in l(x, t)} h(y, t) \mathrm{d} y \mathrm{~d} x .
$$

\section{Measurement and characterisation of longitudinal size and shape}

Fig. 4A shows the relationship between the volume and the width of the plasmodia. For the larger plasmodia $\left[V>0.1 \mathrm{~mm}^{3}\right]$, the maximum width was independent of the volume because their bodies were laterally confined by the narrow lane. To quantify the longitudinal size, which is independent of the width, we estimated the volume per unit width, $V_{l}(t)$ $\left(\mathrm{mm}^{3} \mathrm{~mm}^{-1}\right)$, which is calculated as volume/mean width $V_{l}(t)=V l$ $w_{\text {mean }}(t)$ (Fig. 4B)

We refer to the contour of thickness distribution of the plasmodium in the longitudinal direction as the 'longitudinal shape'. More precisely, on the longitude axis for a unidirectionally migrating plasmodium, the longitudinal shape of the plasmodium at a given time is estimated as $\left\{\left(x, h^{\prime}(x, t)\right) ; x\right.$ is a body point at $t\}$, where $h^{\prime}(x, t)=\max _{y \in l(x, t)} h(y, t)$ is the maximum thickness along the perpendicular axis centred at $x$. The three quantities $x_{\mathrm{L}}, h_{\max }$, $x_{\max }(t)$ were measured to describe the longitudinal shape, where the length $x_{\mathrm{L}}(t)$ is the distance from the leading edge to the tail edge, $h_{\max }(t)\left[=\max _{x} h^{\prime}\right.$ $(x, t)]$ is the maximum thickness and $x_{\max }(t)$ is the distance from the frontal tip to the position of maximum thickness. Whenever the longitudinal shape is described, we use a moving longitude coordinate, the origin of which is always set at the position of the leading edge on the (fixed) longitude axis of the plasmodium; the direction of the longitude axis is reversed such that $x^{\prime}=x_{f}-x$ (Fig. 3B).

\section{Determination of intrinsic period of thickness oscillation $T_{0}$}

It is known that plasmodia show oscillations in thickness with a period of $60-120 \mathrm{~s}$. These oscillations are coordinated along the longitudinal body axis with some phase gradient and produce protoplasmic shuttle streaming (Alexopoulos et al., 1996; Kessler, 1982; Kamiya, 1959). This rhythmic contraction also gives rise to a to-and-fro motion of the leading edge during each cycle. However, although the oscillations are often regarded as 'regular oscillation', the period of the oscillation in each plasmodium is not strictly constant, but is variable in time (Coggin and Pazun, 1996). In this paper, using spatiotemporal thickness data along the longitudinal axis $h(x, t)$, we estimated the intrinsic period of the thickness oscillation for each time $t, T_{0}(t)$. A more complete description is given in the Appendix.

\section{Averaging of variables over intrinsic period of thickness oscillation}

The regular oscillation in thickness may induce oscillation in various other variables including the longitudinal shape and the locomotion speed with the same intrinsic period. To get rid of this short-term, regular variation from 

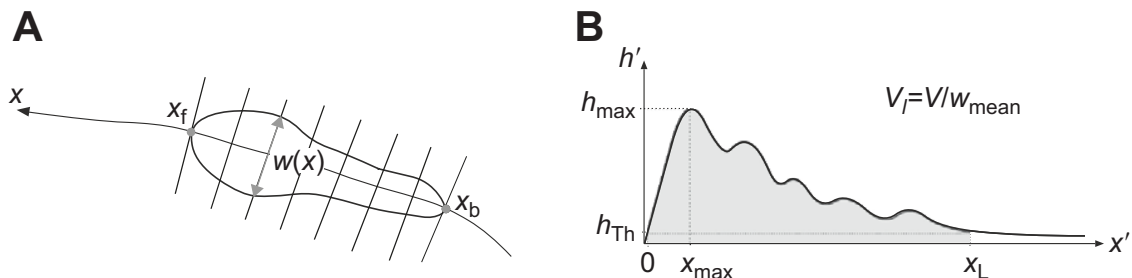

Fig. 3. Coordinate system and longitudinal shape. (A) Longitudinal coordinate and its perpendicular axis. (B) Characterisation of longitudinal shape of plasmodium: $x_{\max }$ denotes the position of the foremost tip, $x_{\mathrm{L}}$ is the effective body length and $h_{\max }$ is the maximum thickness of the frontal part. $h_{\mathrm{Th}}$ is a threshold used to determine the front edge. The abscissa is the longitudinal axis of the moving longitude coordinate at a given time. The ordinate is the thickness of the longitudinal shape. More details are given in the text. Plots are not from real data and are for illustration only.

our further analysis, each variable $z(t) \in\left\{x_{\mathrm{L}}(t), h_{\max }(t), x_{\max }(t), v(t), h^{\prime}(x, t)\right\}$ was averaged over the period $T_{0}(t)$ such that:

$$
z(t)=\int_{s=t-T_{0}(t) / 2}^{t+T_{0}(t) / 2} z(t-s) \mathrm{d} s / T_{0}(t) .
$$

\section{Statistical analysis}

In order to estimate the power-law-type functional relationship between two variables, major axis regression between the log-transformed variables was employed because all the variables were subject to measurement errors in this study (Sokal and Rohlf, 1994). The fitting result is denoted as $y=b x^{a \pm \delta a}$, where $a \pm \delta a$ means that the $95 \%$ confidence interval of $a$ is $(a-\delta a, a+\delta a)$. Moreover, the correlation coefficient $r$ of the variables was estimated by Pearson's method after the $\log$ transformation. The probability value of the $t$-test on $r\left(H_{0}: r=0\right)$ was placed in the text only when the probability was more than $1.0 e-10$. The tail of the longitudinal shape $\left(x_{\max } \leq x \leq x_{2}\right)$ was fitted using the exponential function $\beta \exp \left[-\left(x-x_{\max }\right) / \alpha\right]+\gamma$, where $x_{2}$ is the position at which the thickness is equal to $0.3 h_{\max }$ at that time. The exponent $\alpha$ is referred to as the 'exponential decay distance' here. The semi-logarithmic relationship of the intrinsic period of thickness oscillation $\left(\bar{T}_{0}\right)$ on a longitudinal variable $\left(\bar{V}_{l}, \bar{x}_{\mathrm{L}}, \bar{x}_{\max }\right.$ or $\left.\bar{h}_{\text {max }}\right)$ was estimated by non-linear regression which was performed using the nls package with a port algorithm of version 2.14.2 of R software ( $\mathrm{R}$ Development Core Team, 2012). The correlation coefficient between each longitudinal variable and $\bar{T}_{0}$ was also estimated after the log transformation of the longitudinal variable. The period of the large, long-term variation of a variable $z(t) \in\left\{h_{\max }, T_{0}, v\right\}, T_{1}(z)$, was estimated as follows: the auto-correlation function of the time-series of $z(t)$ was calculated after a band-pass filter (period $=3-40 \mathrm{~min}$ ) was applied. The period was then evaluated as the smallest time-lag at which the auto-correlation function had a positive peak. The time-lag maximising the cross-correlation between $x, y \in\left\{h_{\max }, T_{0}, v\right\}, \tau^{*}(x, y)$, was estimated as follows: the very slow (hourly) oscillatory component (>60 $\mathrm{min}$ ) of each variable was removed by subtracting the mean value averaged over a period $(=30 \mathrm{~min})$. The time-lag $\tau^{*}$ maximising the correlation between $x(t+\tau)$ and $y(\tau)$ was then extracted from the range $-20 \leq \tau \leq 20 \mathrm{~min}$

\section{RESULTS}

\section{Size dependence of longitudinal shape}

Fig. 5A shows examples of the longitudinal shape of plasmodia with three different sizes. Their thickness decreases exponentially towards the rear after the thickness reaches its maximum value in the leading part. Moreover, the exponential decay distance $(\alpha)$ follow the volume per unit width $\left(\bar{V}_{l}\right)$ such that $\bar{\alpha} \propto \bar{V}_{l}^{0.59 \pm 0.04} ; r=0.97$ (Fig. 5B). The exponential decay of the thickness indicates that almost all protoplasm is concentrated in the frontal part. However, we often observed a protuberance in the caudal region, especially in smaller plasmodia.

Fig. 5C shows the longitudinal size dependency of the characteristics $\left(x_{\mathrm{L}}, h_{\max }, x_{\max }\right)$ of the longitudinal shape of the plasmodia, where $x_{\mathrm{L}}$ is the body length, $h_{\max }$ is the maximum thickness and $x_{\max }$ is the distance from the frontal tip to the position of maximum thickness. The following allometric relationships hold: $\quad \bar{h}_{\max } \propto \bar{V}_{l}^{0.40 \pm 0.03} ; \quad r=0.97, \quad \bar{x}_{\max } \propto \bar{V}_{l}^{0.50 \pm 0.04} ; \quad r=0.97$, $\bar{x}_{\mathrm{L}} \propto \bar{V}_{l}^{0.66 \pm 0.02} ; r=0.99$. The growth rate of the body length $\left(x_{\mathrm{L}}\right)$ with respect to the volume per unit width $\left(V_{l}\right)$ is larger than the other two characteristics $\left(x_{\max }, h_{\max }\right)$. This shows that large plasmodia are long and flat, whereas small plasmodia are short and thick (Fig. 5D). The clear allometric relationships above indicate that we can adopt whichever characteristic $\left(V_{l}, x_{\mathrm{L}}, x_{\max }\right.$ or $\left.h_{\max }\right)$ we like to describe the longitudinal size.
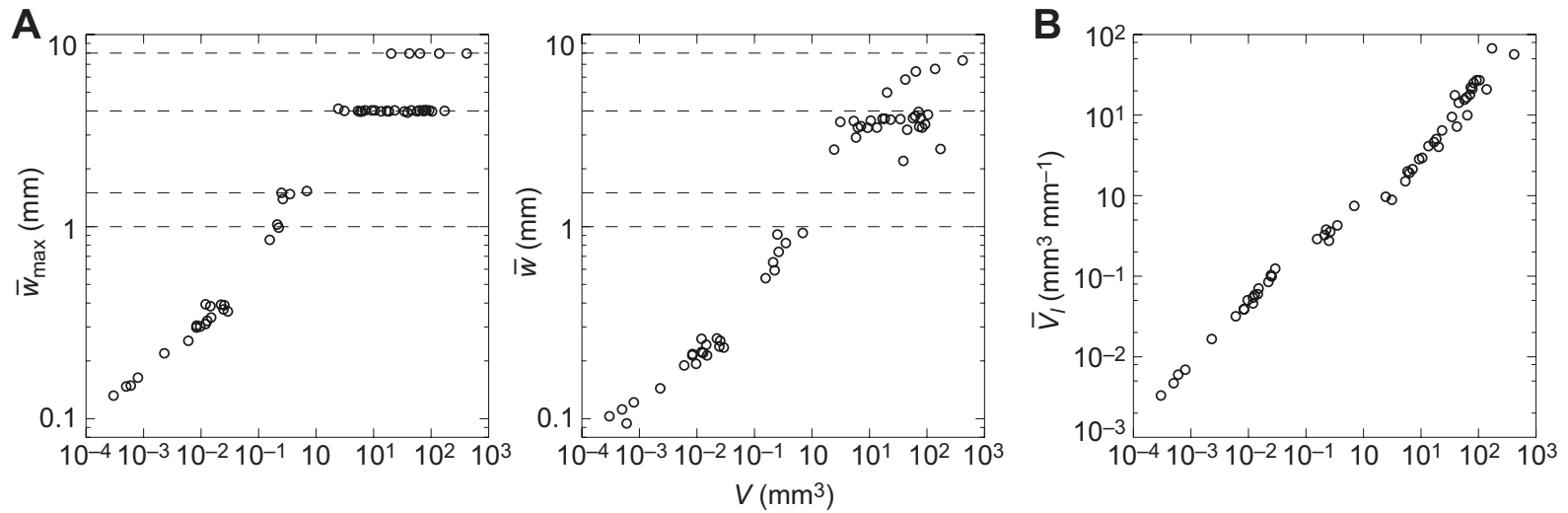

Fig. 4. Dependence of width and longitudinal size on the cell volume. $(A)$ Volume $(V)$ versus the maximal width $\left(\bar{w}_{\text {max }}\right)($ left panel $)$ and the mean width $\left(\bar{w}_{\text {mean }}\right)$ (right panel). The dashed lines represent the width of the lanes for the larger plasmodia. $(C)$ Volume versus volume per unit width $\left(\bar{V}_{l}=V / \bar{W}_{\text {mean }}\right)$. Data are plotted on double log scales. 
A
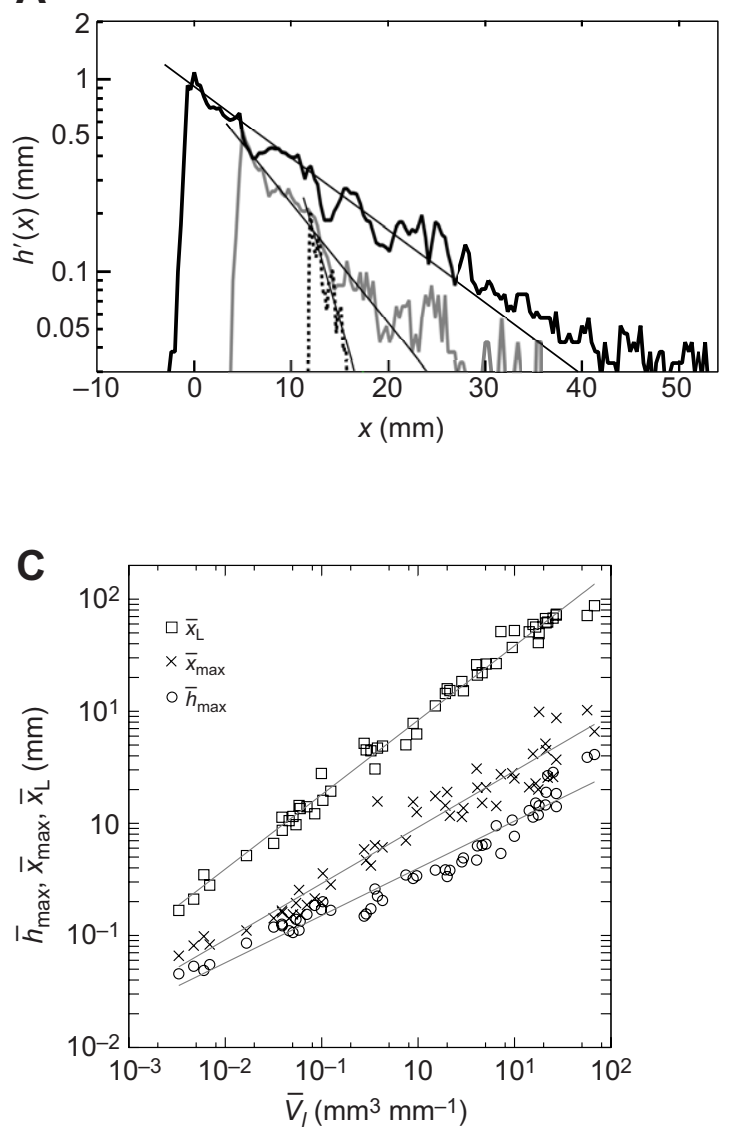

B

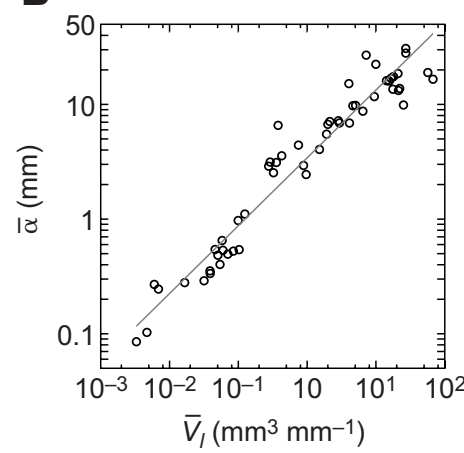

D

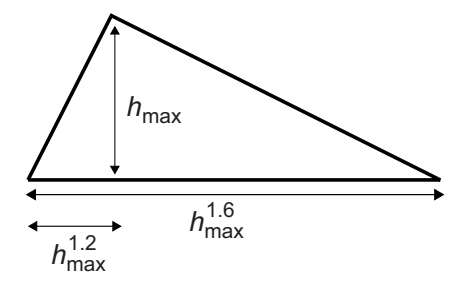

Fig. 5. Size dependence of longitudinal shape. (A) Examples of the longitudinal shape of plasmodia with three different sizes, where the shapes were averaged over the intrinsic periods of the thickness oscillation. Data are plotted on a semi-log scale. Examples for large, medium and small plasmodia are shown by the black solid line, grey solid line and black dotted lines, respectively. The origin of the abscissa axis is set at the position where the longitudinal shape of the largest plasmodium has maximum thickness. The data for the medium and small plasmodia are shifted such that the maximum thickness coincides with that of the large and medium plasmodia, respectively. The lines on the tails represent exponential fits where the decay distances $\alpha$ for the large, medium and small plasmodia are $11.6,6.9,2.6 \mathrm{~mm}$, respectively.

(B) Dependency of the exponential decay distance $\bar{\alpha}$ on the volume per unit width. Data are plotted on a double log scale. Data points fit to the line $\bar{\alpha}=3.42 \bar{V}_{1}^{0}$

(C) Dependence of the longitudinal shape on the volume per unit width. Data are plotted on a double log scale. $\bar{h}_{\max }$ (circles), $\bar{x}_{\max }$ (crosses) and $\bar{x}_{\mathrm{L}}$ (squares) are plotted against $\bar{V}_{l}$. Data points fit the lines $\bar{h}_{\max }=0.40 \bar{V}_{d}^{0.42 \pm 0.03}, \bar{x}_{\max }=0.92 \bar{V}_{l}^{0.50 \pm 0.04}$ $\overline{\mathrm{X}}_{\mathrm{L}}=8.3 \bar{V}_{l}^{0.66 \pm 0.02}$. (D) Summary of scaling property in the longitudinal shape.

\section{Longitudinal size dependency of locomotion speed}

Fig. 6 shows the dependence of locomotion speed $(v)$ on the maximum thickness of the plasmodium. The locomotion speed is almost proportional to the maximum thickness of the plasmodium $\left(\bar{v} \propto \bar{h}_{\max }^{0.94 \pm 0.10}, \quad r=0.93\right)$. The other characteristics also have allometric relationships with the locomotion speed (data points fit to the curve $\bar{v}=0.071 \bar{V}_{l}^{0.39 \pm 0.04}, r=0.94 ; \bar{v}=0.020 \bar{x}_{\mathrm{L}}^{0.59 \pm 0.06}$, $\left.r=0.94 ; \bar{v}=0.076 \bar{x}_{\max }^{0.77 \pm 0.12}, r=0.88\right)$.

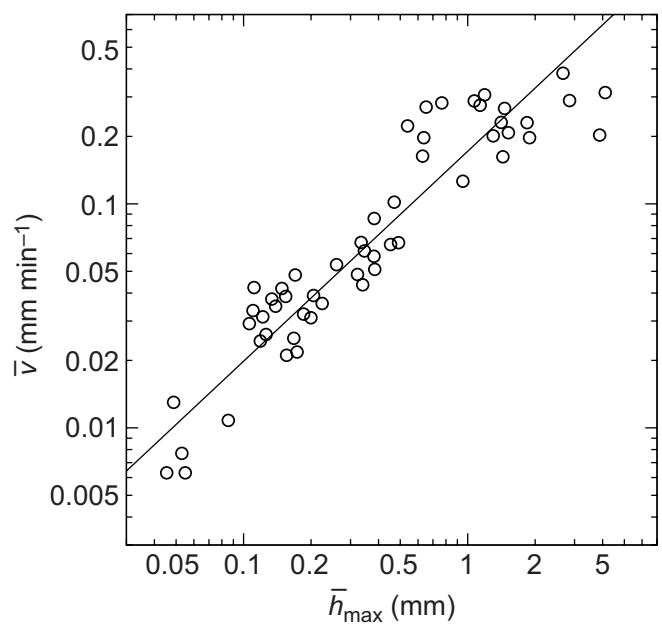

Fig. 6. Longitudinal size dependency of locomotion speed. $\bar{h}_{\max }$ and $\bar{v}$ are plotted on a double log scale. Data points fit to the line $\bar{v}=0.17 \bar{h}_{\max }^{0.94 \pm 0.10}$.

\section{Longitudinal size dependency of intrinsic period $\boldsymbol{T}_{\mathbf{0}}$}

Fig. 7 shows the dependence of the intrinsic period of the thickness oscillation on the maximum thickness of the plasmodium. The period increases logarithmically with the maximum thickness of the plasmodium $\left(\bar{T}_{0}=15.3 \log \left(\bar{h}_{\max }\right)+98.3, r=0.75\right)$. The period also increase logarithmically with the other characteristics $\left(\bar{T}_{0}=6.8 \log \left(\overline{V_{l}}\right)+92.1, \quad r=0.77 ; \quad \bar{T}_{0}=10.0 \log \left(\bar{x}_{\mathrm{L}}\right)+82.9\right.$, $\left.r=0.76 ; \bar{T}_{0}=13.5 \log _{10}\left(\bar{x}_{\max }\right)+92.6, r=0.78\right)$. Our results are

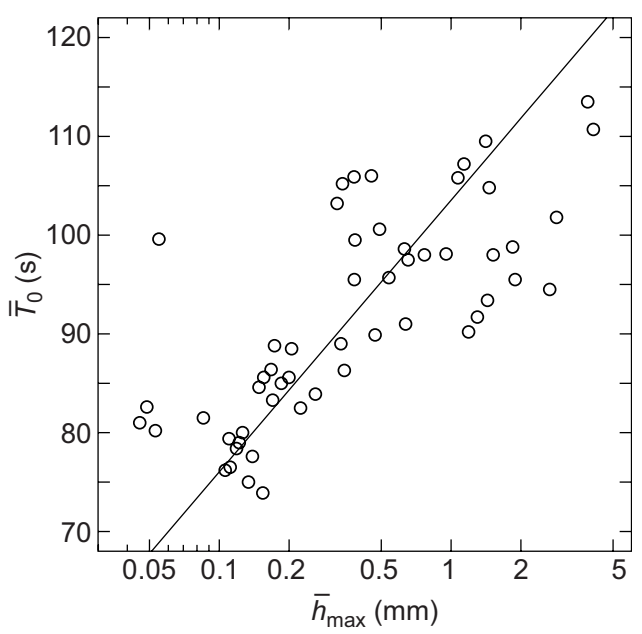

Fig. 7. Longitudinal size dependency of intrinsic period $T_{0} \cdot \bar{T}_{0}$ is plotted on a linear scale, while $\bar{h}_{\max }$ is plotted on a log scale. The solid line is $\bar{T}_{0}=15.3 \log _{10}\left(\bar{h}_{\max }\right)+98.3$, which is given by fitting of the data to log function. 
A

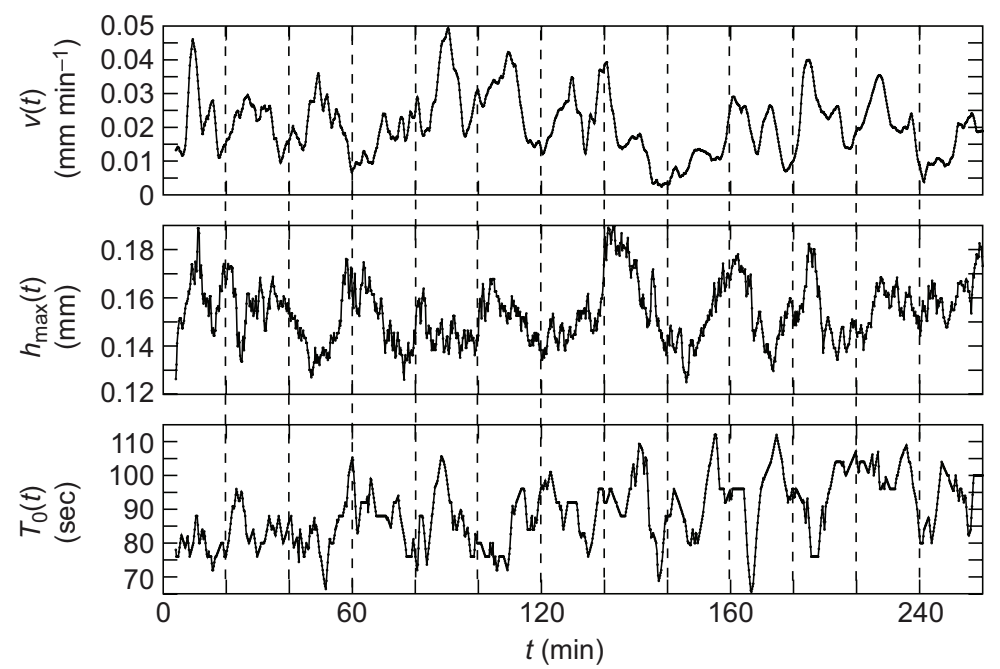

B

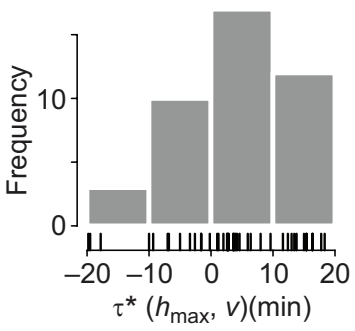

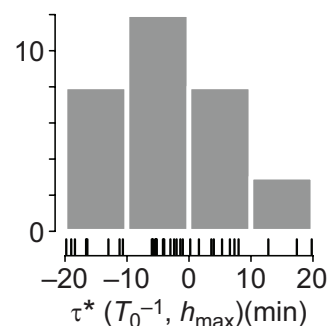

$\tau^{*}\left(T_{0}^{-1}, h_{\max }\right)(\min )$

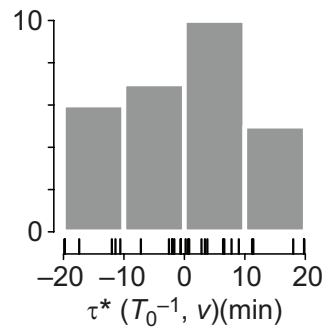

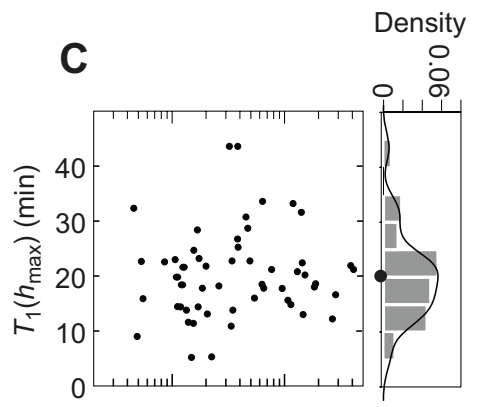
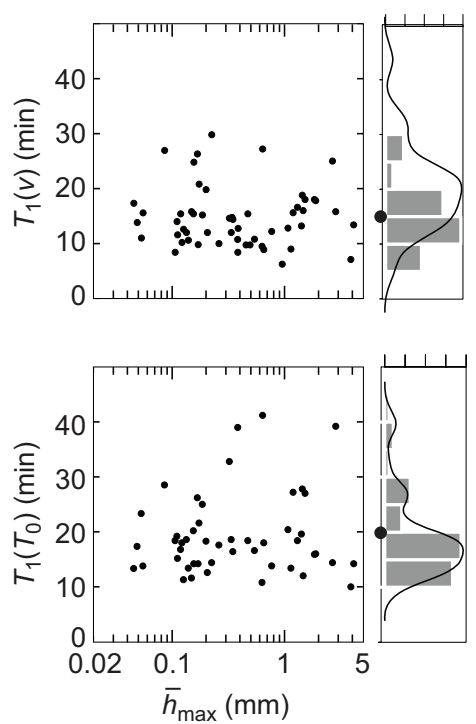

Fig. 8. Large, size-independent long-term variation in speed, shape and intrinsic period. (A) Example of $v(t)\left(\Delta_{t}=8\right.$ min $), h_{\text {max }}(t)$ and $T_{0}(t)$ for a plasmodium (B) Histogram of the time lags maximising the cross-correlation between pairs of parameters among the time-series $T_{0}^{-1}(t), v(t)$ and $h_{\text {max }}(t)$. (C) Size versus period of the large, long-term variation $T_{1}$ of $h_{\max }(t), v(t)$ and $T_{0}(t)$. The abscissa $\left(h_{\max }\right)$ is log scale and the ordinates are linear scale. The correlation coefficients are $r=0.09(P>0.5), r=0.04(P>1.2)$ and $r=0.10(P>0.49)$, respectively. The right-hand panels show histograms of the period $T_{1}$ for each variable. Estimates of kernel density using a Gaussian kernel are superimposed on the histograms. The dot on each ordinate axis indicates the mean of the distribution.

consistent with the previous studies cited above. However, this is the first study involving systematic measurements of plasmodia covering a wide range of sizes during free locomotion.

\section{Long-term large variation in speed, shape and intrinsic period}

During free locomotion, the locomotion speed, longitudinal shape and the intrinsic period of the thickness oscillation always exhibited large variations with a period of a few tens of minutes (Fig. 8A). The oscillations in these parameters often co-varied with fixed time lags, but the phase relationships between them were not identical in the observed plasmodia (Fig. 8B). However, statistically, the change in the locomotion speed $(v)$ tended to proceed the few minutes against that in the longitudinal shape $\left(h_{\max }\right)$ [Fig. 8B, left panel; $\tau^{*}\left(h_{\max }, v\right)=5.0$ min ( $t$-test for $\left.H_{0}: \tau^{*}>0, P<0.0001\right]$. The periods of these longterm oscillations are all independent of the cell size $(r \leq 0.10$, $P>0.49$ ) (Fig. 8C).

\section{DISCUSSION}

\section{Locomotion speed is scaled by the longitudinal size}

We found that the locomotion speed is scaled by the longitudinal size as the length, maximum thickness or volume per unit width rather than by the overall cell size. In other words, the plasmodia migrate faster as the passage gets narrower, which mimics their natural habitats inside rotted trees and underground. This indicates that the self-propelled mechanism of directional movement is confined to the longitudinal section of the body and that the lateral two-dimensional extension is not directly connected to the factors that govern the locomotion. A similar result has been reported for the relationship between locomotion speed and longitudinal size of the pseudoplasmodium (slug) Dictyostelium discoideum (Inouye and Takeuchi, 1979; Rieu et al., 2005). Moreover, the scaling exponent, $\sim 0.6$, between the speed and body length of the plasmodia reported here is a good agreement with that of the slugs on agar plate, $v_{\text {slug }} \propto L_{\text {slug }}^{0.63 \pm 0.06}$, which was obtained by fitting the data points in Inouye and Takeuchi (1979). This relationship also provides a rough estimate for the locomotion speed of organisms with smaller size, which is in the range of $1-3 \mu \mathrm{m} \mathrm{min}{ }^{-1}$ for a $10-30 \mu \mathrm{m}$ length.

It should be noted that the scaling discussed in this study is restricted to almost one-dimensional locomotion and the scaling for two-dimensional migration may differ from the present conclusions. However, even in a two-dimensional migration, if the thickness profile along an extending direction is similar to the longitudinal shape in one-dimensional locomotion, we may also expect the extending speed in that direction to obey the scaling law observed in one dimension. Further investigation of this aspect will be left for future studies. 


\section{Alternative physical models fulfil $v \propto h_{\max }$}

We consider the physico-mechanical meaning of the proportional relationship between the speed and maximum thickness of the plasmodium. Insight can be gained by conducting a dimensional analysis under the following two assumptions. (1) The speed $v$ is proportional to the characteristic length $h(\mathrm{~L}), v \propto h$. (2) The speed $v$ is a function of at most three possible mechanical quantities, including $h$, among the following: $\rho\left(\mathrm{FL}^{-4} \mathrm{~T}^{2}\right)$ (density), $\mu\left(\mathrm{FL}^{-1} \mathrm{~T}\right)$ (viscosity), $p\left(\mathrm{FL}^{-2}\right)$ ( pressure in the cell), $\tau\left(\mathrm{FL}^{-1}\right)$ (contractile and tensile force of ectoplasmic cortex per unit arc length) and $D(\mathrm{~F})$ (drag force with the substratum), where the contractile and tensile force includes the active force generated by actomyosin fibrils in the cortex and the passive force due to gel-like material characteristics of the cortex. Here, three is the smallest number of variables that can represent $v$ as their function, because the number of related fundamental physical units $(\mathrm{F}, \mathrm{L}, \mathrm{T})$ is three (Buckingham $\pi$ theorem, see McMahon and Bonner, 1983). These assumptions lead to the functional relationship $v=$ constant $\times p h / \mu$ as a unique solution.

We now attempt to obtain a migration model for plasmodium that satisfies the above functional relationship. For simplicity, we can regard a plasmodium as a dome-shaped ectoplasmic cortex with radius $h=h_{\max }$ that envelops the protoplasmic sol (a highly viscous fluid), and we can assume that the flow velocity $v$ is identical to the locomotion speed of the plasmodium. The cortex induces the protoplasmic sol flow due to the pressure difference $p$ generated by the effective surface tension $\tau$, where $p$ is proportional to $\tau / h$ (Laplace pressure; see de Gennes et al., 2004). By the Stokes equations, the pressure gradient $p / h$ and the viscosity term $\mu v / h^{2}$ balance and thus we have: $v \propto(p / \mu) h=\tau / \mu$. Therefore, the following two alternative situations are implied: (i) if the viscosity coefficient $\mu$ is independent of $h, \tau=\tau(h) \propto h$ is followed by the constraint $v \propto h$. That is, the contraction force generated by the cortex is proportional to the maximum thickness of the plasmodium. This situation is probable if the thickness of the cortex is proportional to the maximum thickness of the plasmodium and also to the contraction force. However, (ii) if $\tau$ is independent of $h$, then $\mu=\mu(h) \propto h^{-1}$. This requires that the viscosity of the frontal part of small plasmodia should be larger than that of large plasmodia. One feature that may give rise to size dependency of the 'effective' viscosity is the channel structure that is observed in the frontal tip of plasmodia (see Matsumoto et al., 2008 for small plasmodia). Further analysis is again left for future studies.

\section{Two examples of the body shape evolution related to size- independent, long-term oscillation}

It is reasonable to ask about the origin of the difference between the scalable and the non-scalable properties over several orders of magnitude. Because the size-dependent properties result from physico-mechanical processes, they are likely to be quite general
A

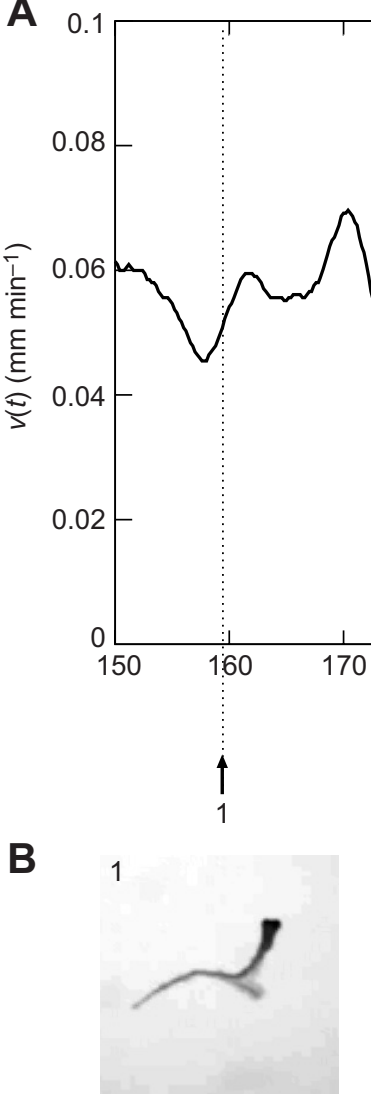

2

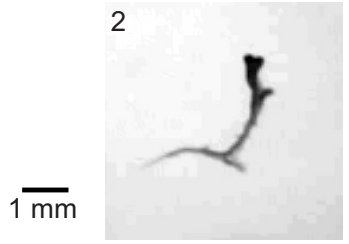

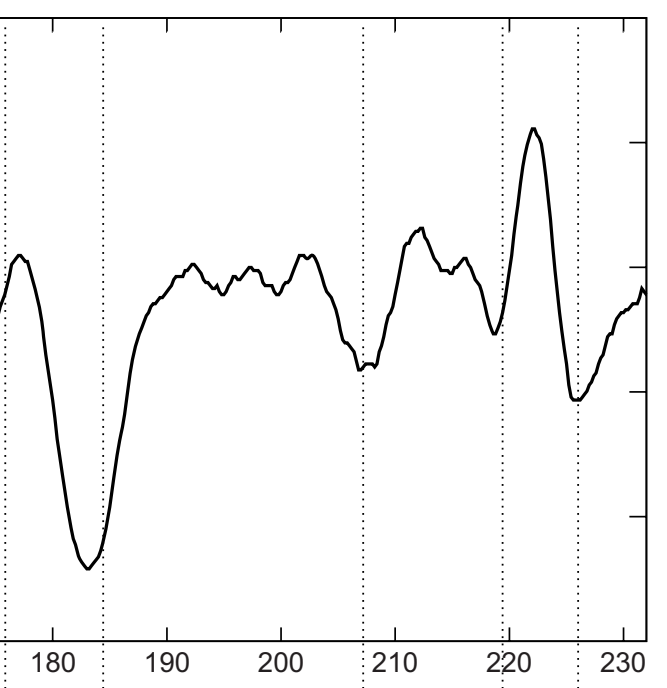

$t(\min )$
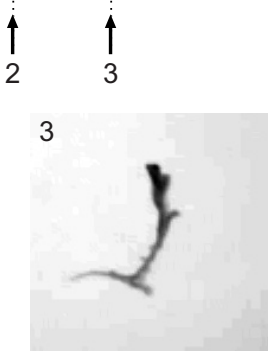

4

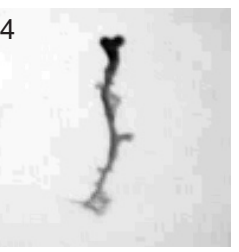

Fig. 9. Branching of the frontal part in the slow-down phase. (A) Locomotion speed $v\left[\mathrm{~mm} \mathrm{~min}^{-1}\right]\left(\Delta_{t}=4 \mathrm{~min}\right)$ of a plasmodium of intermediate size $\left(0.2 \mathrm{~mm}^{3}\right)$ during free locomotion in the Petri dish $(9 \mathrm{~cm}$ in diameter). The timings at which branching of the frontal part was observed are labelled from 1 to 6 . (B) Images recorded at the six timings indicated in $A$. The plasmodium was illuminated from below and the transmitted light images were captured from above; details of the measurement are given in the text. Darker contrast indicates areas with relatively thicker plasmodium. The branching frontal part is observed in the upper region of each image as a dark expanded area. 


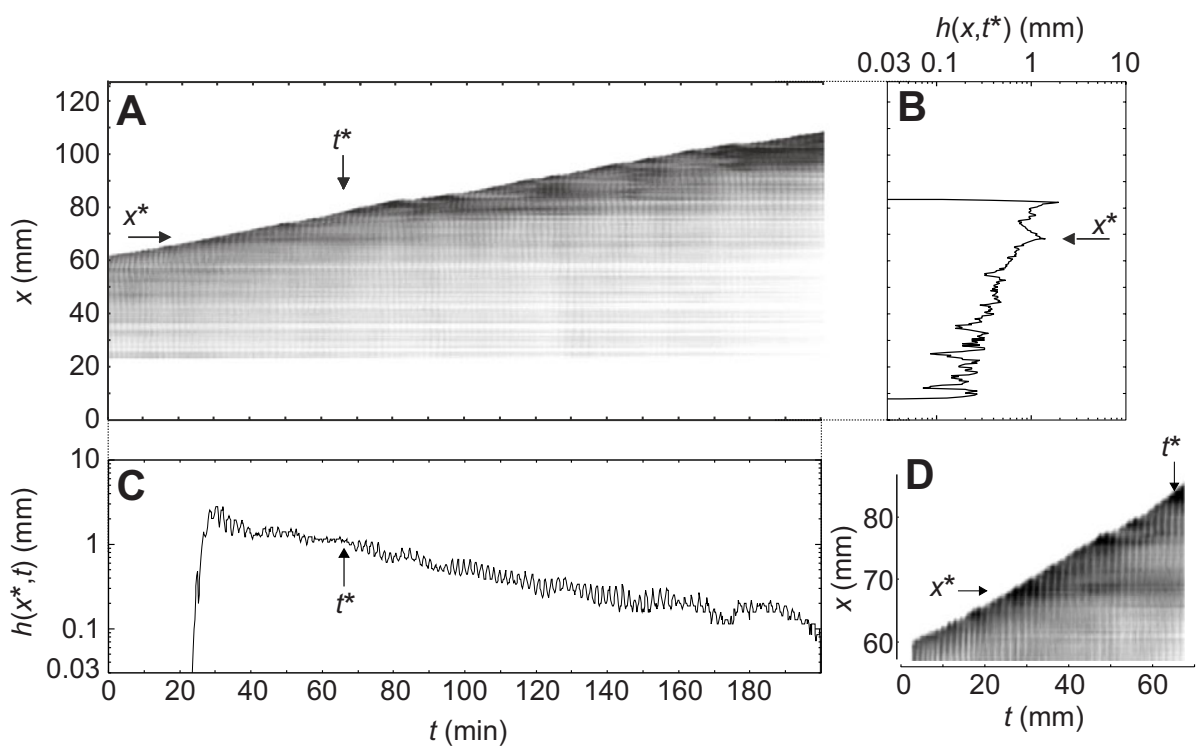

Fig. 10. Undulations in the longitudinal shape. (A) Space-time plot for a plasmodium extending in one dimension towards the upper direction on a plain agar gel lane. Darker contrast indicates areas with relatively thicker plasmodium. The parameters $x^{*}$ and $t^{*}$ are examples of places and times where the thickness of the frontal part is much larger than the mean value. (B) Thickness distribution along the longitudinal direction at time $t^{*}$. (C) Time trace of the thickness of the plasmodium at position $x^{*}$, which exponentially decreases with time. (D) Magnified view of this plot in the region containing $x^{*}$ and $t^{*} . h$ axes are plotted on a log scale, whereas $t$ and $x$ axes are plotted on a linear scale. properties that hold for a wide range of organisms. In contrast, one probable reason is that the size-independent properties result from biochemical processes because chemical reaction speed mainly depends not on the body size itself but on the chemical characteristics and the concentration of the substances involved.

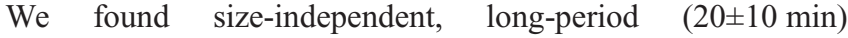
oscillations including speed, shape and intrinsic thickness oscillation period. In order to consider their physiological meanings, we present two examples of body shape evolution tightly coupled with this oscillation.

\section{Branching of the frontal tip}

Under free locomotion on a large enough area with no narrow lane, branching of the frontal tip was sometimes observed in plasmodia of intermediate size. These plasmodia are too large to maintain the tadpole-like shape throughout the measurement period but are not pronounced enough to form a two-dimensional fan-like sheet structure. Fig. 9 shows an example of a time course of the locomotion speed for such a plasmodium $\left(0.2 \mathrm{~mm}^{3}\right)$ during free locomotion. The times at which branching occurred are indicated on the time course; branching was observed shortly after the slowdown phase of the locomotion speed. After each branching event, one of the two advancing fronts stopped extending and disappeared by the time of the next branching event.

\section{Thickness undulation along the longitudinal direction}

Fig. 10A shows a space-time plot of a plasmodium migrating onedimensionally in the upper direction on an agar lane. The thickness of the frontal tip varied with a period of approximately $10 \mathrm{~min}$. This long-term oscillation is visible from the stripe pattern parallel to the time axis. In contrast, the stripe pattern parallel to the spatial axis arises from the shorter thickness oscillation with an intrinsic period. The labels $x^{*}$ and $t^{*}$ in the figure indicate examples of places or times where the thickness of the frontal part became much larger
A
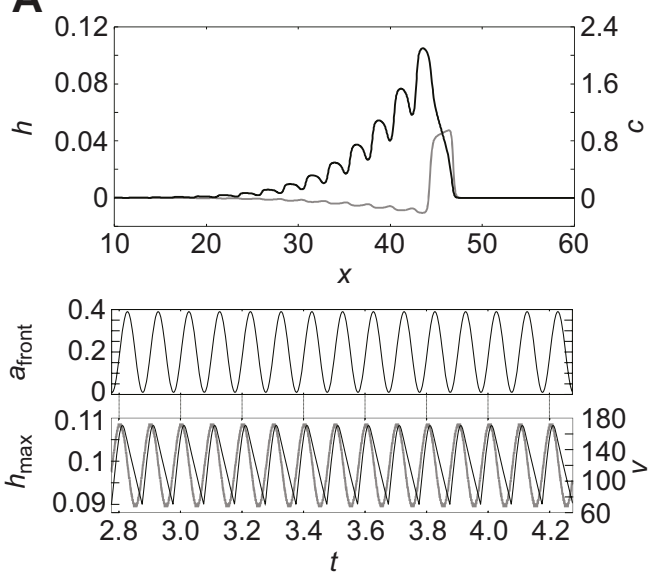

B

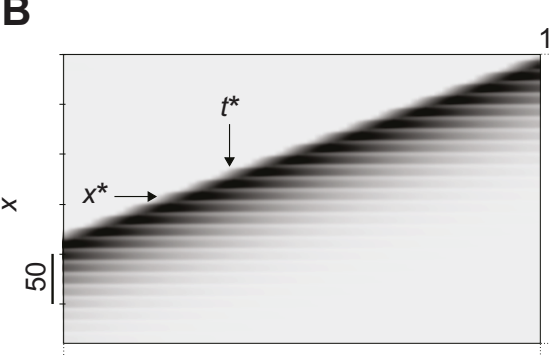

$h\left(x, t^{*}\right)$
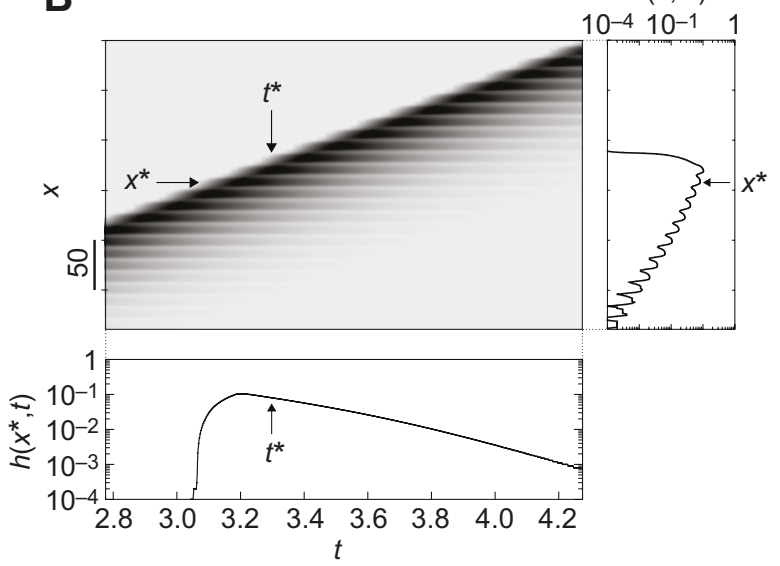

Fig. 11. Propagating wave solution in the reaction-diffusion model with periodic modulation of activity level in the frontal region. (A) Top panel is a snapshot of the propagating wave solution which moves towards the right. The black and grey lines indicate the body thickness $h$ and the chemical concentration $c$, respectively. Middle panel shows a time course of the activity level in the frontal region $X_{\text {front }}$, given by $a_{\text {front }}(t)=a(x, t)=0.2+0.19 \sin (20 \pi t)$, where $X_{\text {front }}(t)$ is a

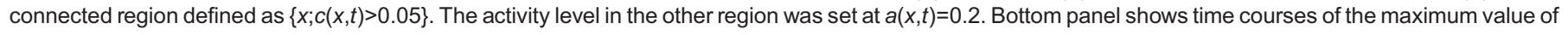
$h$ (black line) and the propagation speed, $v\left(\Delta_{t}=0.5\right)$, of the wave (grey line), which was calculated from $X_{\text {front }}(t)$. (B) Space-time plot of the value of $h(x, t)$

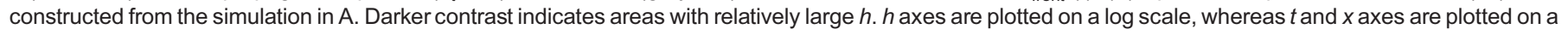
linear scale. No transient motion was exhibited. 
than the mean value, indicated by the relatively dark contrast. The time trace of the thickness at a given position decreases exponentially with time (Fig. 10C). Fig. 10B shows the thickness distribution in the longitudinal direction at a given time, large fluctuations are superimposed on the base line which exponentially decreases from the maximum thickness at the frontal tip. We note that similar fluctuations can be observed in Fig. 5A. Each undulation in the tail corresponds to a peak in the long-term, large variation of thickness of the frontal tip; this correspondence gives rise to the stripe pattern parallel to the time axis in the space-time plot (Fig. 10A).

Rodiek et al. (2015) recently reported two distinct patterns of intracellular thickness oscillations along the longitudinal body axis depending on the locomotive speed of the freely migrating small tadpole-shaped plasmodium. Interestingly, one of them, described as having a multinodal standing wave-like pattern, seems to have a similar spatial structure with the above-mentioned thickness undulation pattern. That is, it has a large amplitude oscillatory component with a relatively long period $(\sim 10 \mathrm{~min})$ and a fairly regular wavelength, which is similar to the migratory distance during the period.

\section{Physiological role of the size-independent, long-term large oscillation}

Our observations indicate that plasmodia commonly have an internal rhythm with a size-independent period that appears in many aspects of the organism. It is useful to consider the physiological function of this rhythm in the plasmodium. One of the important aims of the organism during free locomotion is exploitation of the surroundings. Thus, the long-term modulation of speed determines a spatial sampling size of the environment, because in the slower phase, the organism stays in a given area for longer or is afforded a chance to change direction by branching. However, the locomotion speed may be largely modulated by various external signals such as changes in the temperature, moisture, light level or chemical substances. Therefore, exposure to such signals during locomotion might be recorded in the form of thickness undulations in the longitudinal direction. This can be envisaged as a kind of dynamic encoding of temporal information to the spatial structure. Moreover, if the branching in small plasmodia is related to the vein network structure of large plasmodia with a two-dimensional fan-like shape, the events experienced are also recorded in the form of a two-dimensional mesh pattern in large plasmodia. Therefore, if the one- and two-dimensional body shape at a given time represents a sequence of episodic events, the shape may influence the future behaviour of the organism. The body shape of plasmodia could thus be considered as a prototype of episodic memory.

\section{Simple mathematical model for cell locomotion with thickness undulation}

In order to explore possibilities for the dynamic encoding of biochemical activities into the body shape of the plasmodium, we constructed a mathematical model for cell locomotion. The exponential decay of the thickness indicates that almost all protoplasm is concentrated in the frontal part. Takagi et al. (2007) have reported that a frontal tip amputated from a plasmodium during one-dimensional locomotion can migrate for some time at the same speed as before without the regular thickness oscillation. This selfmigration of the amputated frontal tip is evidence that migration ability can be localised at the frontal tip. Furthermore, the fact that the migration lasts for only a short time (Takagi et al., 2007) suggests that the engine in the frontal tip is a self-organised local structure that is dynamically and stably maintained in the plasmodium system.

It has also been reported that the polarised intracellular chemical pattern corresponds to the polarity of the plasmodium; for example, the ATP concentration is higher at the locomotion front and becomes lower towards the tail (Mori et al., 1986; Ueda et al., 1987). According to these facts, we can consider the locomotion of plasmodium as the propagation of a polarised protoplasm pulse based on an excitable system that can be expressed as a onedimensional reaction-diffusion system with two variables, $c$ and $h$ (Takagi et al., 2007; Ueda et al., 2011):

$$
\begin{gathered}
c_{t}=D_{\mathrm{C}} c_{x x}+f(c, h ; a), \\
h_{t}=D_{h} h_{x x}+\epsilon g(c, h) .
\end{gathered}
$$

Here, $c=c(x, t)$ is the concentration of a chemical $C$ that is responsible for the sol-gel transformation, $h=h(x, t)$ is the thickness of the body, $a$ is the activity level of $C, x$ is a coordinate along the longitudinal direction, $D_{\mathrm{C}} \gg D_{h}, \varepsilon \ll 1$ which implies that $\tau_{c} \ll \tau_{h}$ where $h$ and $c$ are slow and fast variables, respectively. We assume that this is an excitable system where the parameter $f$ gives the bi-stability for $c$, and $h$ is a slow variable. For simplicity, we adopt $f(c, h ; a)=c(1-c)$ $(c-a)-h$ and $g(c, h)=c-h$, according to a FitzHugh-Nagumo type system. This system has a travelling wave solution with speed $v(\varepsilon)$. As $\varepsilon \rightarrow 0$, the speed approaches $v^{*}=\sqrt{2 D_{\mathrm{C}}}[(1 / 2)-a]$ (Kawahara and Tanaka, 1983). This implies that the speed of the wave front is linearly dependent on the activity level $a$ and on the square root of the diffusion level $D_{\mathrm{C}}$ of the chemical. We now extend the model by introducing a slow modulation of the activity level $a$ of the sol-gel transformation only in the frontal region of the wave. We assume this modulation results from unknown biochemical processes that are independent of the body size. Fig. 11 shows a numerical result of the model where a propagating wave with an oscillatory wave front and an undulating tail was obtained. Here, the oscillation of the activity level in the frontal part induces an oscillation of the thickness there (Fig. 11A, bottom panel). The propagation speed of the waves is also temporally modulated as expected from the exact calculation of the speed of the wave above in the case of constant activity level (Fig. 11A, bottom panel). Fig. 11B shows a spacetime plot of a propagating solution. The thickness $h$ modulation in the frontal tip remains in the form of undulations superimposed on the wave whose baseline exponentially decreases with time or distance from the frontal part. A similar profile is shown in Fig. 10A for a plasmodium. However, our understanding of physiological basis, especially the physiological origin of the slow modulation, is still poor. Further studies should be performed to verify this hypothesis.

\section{Appendix}

\section{Determination of intrinsic period of thickness oscillation $\boldsymbol{T}_{\mathbf{0}}$}

Suppose that $x_{f}(t)$ and $x_{b}(t)$ are the positions of the leading edge and tail edge on the longitudinal axis at time $t$, respectively, as described in the 'Data analysis' section. Taking the moving coordinate $u$ with its origin always at the leading edge, we obtained the time series of the thickness, $h_{u}(t)=h\left[x_{f}(t)-u, t\right]$, at each relative position $u \in\left[0, u_{b}\right]$, where $u_{b}=\min _{t}\left\{x_{f}(t)-x_{b}(t)\right\}$ is the minimum tail edge of the plasmodium. We now have $u_{b} / \delta x(=M)$ time series for each plasmodium, where $\delta x$ is a pixelated spatial unit. After respective time series were normalised in zero mean and unity standard deviation, the $M$-dim time series $\left\{h_{u}(t)\right\}$ was projected on the principal components (PCs), and reduced to $M^{\prime}$-dim time 
series $\left\{h_{j}^{\prime}(t) ; j=1, \ldots, M^{\prime}\right\}$ where $M^{\prime}$ is the minimal number such that the cumulative contribution ratio up to the $M^{\prime}$-th PC is larger than $70 \%$. To obtain the oscillatory component with periods in the range of a few tens of seconds to a few minutes, the thickness variation $\Delta h^{\prime}{ }_{j}(t)$ at each $j$ was derived by moving-averaging with the time-window $30 \mathrm{~s}$ (low-pass filtering) and subtracting the moving-averaged component with the time-window $\bar{T}^{(0)}$ (high-pass filtering), where $\bar{T}^{(0)}$ is a initial putative mean period $=80 \mathrm{~s}$. By extracting the peak times $\left\{t_{j, n} ; n=1, \ldots, N(j)\right\}$ of $\Delta h^{\prime}{ }_{j}(t)$, we obtained a series of the oscillation period at each $j$, $T_{j}^{(1)}\left(t_{j, n+(1 / 2)}\right)(n=1, \ldots, N(j)-1)$, where $t_{j, n+(1 / 2)}=\left(t_{j, n+1}+t_{j, n}\right) / 2$. Then we obtained a new putative mean period $\bar{T}^{(1)}$ by averaging $\left\{T_{j}^{(1)}\left(t_{j, i}\right)\right\}$ over $j$ and $t_{j, i}$ after the removal of outliers. Moreover, a time series for the oscillation period $T_{j}^{(1)}(t)$ at each $j$ was reconstructed by linear interpolation of $T_{j}^{(1)}\left(t_{j, i}\right)$ along the time axis. To obtain a more reliable estimation of the oscillation period, we iterated the above procedure twice after replacing $\bar{T}^{(0)}$ with $\bar{T}^{(1)}$ and obtained $\bar{T}^{(3)}$ and $\left\{T_{j}^{(3)}(t)\right\}$. Finally, $\bar{T}^{(3)}$ and $T^{(3)}(t)$ was adopted as the mean intrinsic period $\bar{T}_{0}$ and the time series of the intrinsic period $T_{0}(t)$ of the plasmodium, where $T^{(3)}(t)$ was given by average $\left\{T_{j}^{(3)}(t)\right\}$ over $j$ after the removal of outliers.

\section{Acknowledgements}

S.K. acknowledges helpful comments about dimensional analysis from Dr Yoshimi Tanaka.

\section{Competing interests}

The authors declare no competing or financial interests.

\section{Author contributions}

S.K.: conception and design, acquisition of data and analysis and interpretation of data, mathematical modelling, drafting the article, critically revising the draft. S.T.: analysis and interpretation of data. T.N.: critically revising the draft. T.U.: conception and design, critically revising the draft. All authors gave final approval of the version to be published.

\section{Funding}

This work was supported by the Strategic Japanese-Swedish Research Cooperative Programme from JST and by JSPS KAKENHI (grant no. 26310202), a Grant-in-Aid for Scientific Research on Innovative Areas 'Fluctuation and Structure' (no. 25103006) and 'Cross talk between moving cells and microenvironment' (no. 25111726) from Mext Japan.

\section{References}

Akitaya, T., Ohsaka, S., Ueda, T. and Kobatake, Y. (1985). Oscillations in intracellular ATP, CAMP and cGMP concentration in relation to rhythmical sporulation under continuous light in the myxomycete Physarum polycephalum. J. Gen. Microbiol. 131, 195-200.

Alexopoulos, C., Mims, C. and Blackwell, M. (1996). Introductory Mycology. New York: John Wiley and Sons Inc.

Ashworth, J. and Dee, J. (1975). The Biology of Slime Moulds. London: Edward Arnold.

Barenblatt, G. (2003). Scaling. Cambridge: Cambridge University Press

Calder, W., III. (1996). Size, Function, and Life History. New York: Dover.

Coggin, S. J. and Pazun, J. L. (1996). Dynamic complexity in Physarum polycephalum shuttle streaming. Protoplasma 194, 243-249.

de Gennes, P.-G., Brochard-Wyart, F. and Quere, D. (2004). Capillarity and Wetting: Phenomena Drops, Bubbles, Pearls, Waves. New York: Springer.
Inouye, K. and Takeuchi, I. (1979). Analytical studies on migrating movement of the pseudo-plasmodium of Dictyostelium discoideum. Protoplasma 99, 289-304.

Ishigami, M., Kuroda, K. and Hatano, S. (1987). Dynamic aspects of the contractile system in Physarum plasmodium. III. Cyclic contraction-relaxation of the plasmodial fragment in accordance with the generation-degeneration of cytoplasmic actomyosin fibrils. J. Cell Biol. 105, 381-386.

Kamiya, N. (1959). Protoplasmic streaming. Protoplasmatologia 8, 1-199.

Kawahara, T. and Tanaka, M. (1983). Interactions of traveling fronts: an exact solution of a nonlinear diffusion equation. Phys. Lett. A 97, 311-314.

Kessler, D. (1982). Cell Biology of Physarum and Didymium, Vol. 1, chapter Plasmodial Morphology and Motility, pp. 145-208. New York: Academic Press

Matsumoto, K., Takagi, S. and Nakagaki, T. (2008). Locomotive mechanism of Physarum plasmodia based on spatiotemporal analysis of protoplasmic streaming. Biophys. J. 94, 2492-2504.

McMahon, T. and Bonner, J. (1983). On Size and Life. New York: Scientific American Library.

Mori, Y., Matsumoto, K., Ueda, T. and Kobatake, Y. (1986). Spatio-temporal organization of intracellular ATP content and oscillation patterns in response to blue light by Physarum polycephalum. Protoplasma 135, 31-37.

R Development Core Team. (2012). R: A Language and Environment for Statistical Computing Vienna, Austria. ISBN 3-900051-07-0, http://www.r-project.org

Rasband, W. (1997-2011). Imagej. Bethesda, MD, USA: U.S. National institute of health. http://imageJ.nih.gov/ij.

Rieu, J.-P., Barentin, C., Maeda, Y. and Sawada, Y. (2005). Direct mechanical force measurements during the migration of Dictyostelium slugs using flexible substrata. Biophys. J. 89, 3563-3576.

Rodiek, B., Takagi, S., Ueda, T. and Hauser, M. J. B. (2015). Patterns of cell thickness oscillations during directional migration of Physarum polycephalum. Eur. Phys. J. 44, 349-358.

Schmidt-Nielsen, K. (1984). Scaling: Why is Animal Size so Important. Cambridge: Cambrigde University Press.

Shingleton, A. (2010). Allometry: the study of biological scaling. Nat. Educ. Knowledge 1, 2.

Sokal, R. and Rohlf, F. (1994). Biometry: The Principles and Practice of Statistics in Biological Research. New York: W. H. Freeman and company.

Takagi, S. and Ueda, T. (2008). Emergence and transitions of dynamic patterns of thickness oscillation of the plasmodium of the true slime mold Physarum polycephalum. Physica D 237, 420-427.

Takagi, S., Nishiura, Y., Nakagaki, T., Ueda, T. and Ueda, K.-I. (2007). Indecisive behavior of amoeba crossing an environmental barrier. In Topological Aspects of Critical Systems and Networks (ed. K. Yakubo, H. Amitsuka, G. Ishikawa, K Machino, T. Nakagaki, S. Tanda, H. Yamada and N. Kichiji), pages 86-93. Singapore: World Scientific.

Ueda, T. (2006). An intelligent slime mold: a self-organizing system of cell shape and information. In Networks of Interacting Machines: Production Organization in Complex Industrial Systems and Biological Cells, volume 3 of World Scientific Lecture Notes in Complex Systems, chapter 9 (ed. D. Armbruster, K. Kaneko and A. Mikhailov), pp. 221-245. Singapore: World Scientific.

Ueda, T. and Kobatake, Y. (1980). Contraction rhythm in the plasmodium of Physarum polycephalum: dependence of the period on the amplitude, temperature and chemical environment. Eur. J. Cell Biol. 23, 37-42.

Ueda, T. and Kobatake, Y. (1982). Initiation, development and termination of contraction rhythm in plasmodia of myxomycete Physarum polycephalum. J. Theor. Biol. 97, 87-93.

Ueda, T., Terayama, K., Kurihara, K. and Kobatake, Y. (1975). Threshold phenomena in chemoreception and taxis in slime mold Physarum polycephalum. J. Gen. Physiol. 65, 223-234.

Ueda, T., Mori, Y. and Kobatake, Y. (1987). Patterns in the distribution of intracellular ATP concentration in relation to coordination of amoeboid cell behavior in Physarum polycephalum. Exp. Cell Res. 169, 191-201.

Ueda, K.-I., Takagi, S., Nishiura, Y. and Nakagaki, T. (2011). Mathematical mode for contemplative amoeboid locomotion. Phys. Rev. E 83, 021916.

Vouk, V. (1910). Untersuchungen über die bewegungen der plasmodien I. teil die rhythmik der protoplasmaströmung. Sitzungsber. Kais. Akad. Wissensch. Wien, math.-naturwiss. KI. 119, 853-876. 